\title{
Precessional damping of Fe magnetic moments in a FeNi film
}

\author{
Stefan Buschhorn, Frank Brüssing, Radu Abrudan, and \\ Hartmut Zabel \\ Institut für Experimentalphysik/Festkörperphysik, \\ Ruhr-Universität Bochum, 44780 Bochum, Germany \\ E-mail: stefan.buschhorn@ruhr-uni-bochum.de
}

\begin{abstract}
We report on the element-resolved precessional dynamics of Fe magnetic moments in a homogeneous FeNi thin film. In our pump-probe experiment the magnetic system is excited by a magnetic field pulse from a stripline. The instantaneous response to the field pulse excitation is monitored as a function of time in a stroboscopic measurement using element selective X-ray Resonant Magnetic Scattering (XRMS). Our data show that $\mathrm{Fe}$ and $\mathrm{Ni}$ moments are aligned parallel to each other at all times, while they oscillate around the effective field direction given by the step field pulse and applied bias field. The field dependence of the precessional motion and damping of Fe magnetic moments is analyzed and compared to time-resolved Magneto-Optical Kerr Effect (tr-MOKE) measurement data from literature, showing good agreement.

Additional studies proove the capability of our setup to conduct temperature dependent studies. In case of the presented FeNi system no changes in the frequency or damping behavior are observed within a temperature range of $150-350 \mathrm{~K}$.
\end{abstract}

Submitted to: J. Phys. D: Appl. Phys. 


\section{Introduction}

The studies of magnetization dynamics in ferromagnetic materials provide valuable information about fundamental material properties, which is complementary to or sometimes even more extensive than the information gained from magnetic hysteresis measurements. Whenever the mean magnetization experiences a torque induced by a non-collinear alignment between the effective field and the magnetization vector, a precessional motion around the effective field direction occurs and is accompanied by damping which forces the magnetization vector into the field direction $[1,2]$. The damping of this precession is important for the speed of domain wall propagation and determines the timescale and stability of precessional switching at which future spintronic devices will be based on $[3,4]$. In fact, both switching mechanisms, i.e., domain wall propagation and precessional switching, find their applications in different systems, like bit-patterned media [5] or the race-track memory [6].

The eigen-frequency and the damping constant of a ferromagnetic layer can be determined from ferromagnetic resonance experiments (FMR) [7]. However, in ferromagnetic alloys or multilayer systems conventional FMR does not allow to distinguish directly between the dynamics of the constituent elements. Therefore, FMR of magnetic heterostructures and multilayers may become difficult to analyze if several resonance lines are located very close. Often, the experimental results need to be supplemented by simulations where certain assumptions are made to model the underlying physical mechanisms and the resulting spectra are compared to the measured ones. This lack of element sensitivity has been overcome by performing time resolved Xray Magnetic Circular Dichrosim (XMCD) measurements while the sample is exposed to resonant microwave radiation, be it with a phase-locked [8] or time-averaged [9] technique. In addition to FMR-based techniques, pump-probe setups were realized in recent years. Time-resolved XMCD experiments in reflection or transmission geometry $[10,11]$ provide additional information about the constituting elements while using magnetic field pulse excitation. Each of the element-resolved experimental techniques has specific advantages and disadvantages, which have been described in some detail by Guan et al. [12]. We use time-resolved X-ray Resonant Magnetic Scattering (tr-XRMS) after pulse excitation [13]. This method allows to obtain structural information via resonant magnetic x-ray reflectivity measurements in combination with time-resolved dynamical experiments on one and the same sample under the very same sample conditions. Here we present results on a permalloy $\mathrm{Fe}_{20} \mathrm{Ni}_{80}(\mathrm{Py})$ thin film. In recent years the precessional damping of Py has been investigated in much detail in pumpprobe experiments, including the thickness dependence [14], interlayer effects [15] and vector-resolved experiments [16]. Bailey et al. [10] have investigated the element-specific dynamics of Py, however the authors do not comment on the damping of the individual elements, which is of utmost importance for applications.

We measured the element-resolved magnetization dynamics in a $25 \mathrm{~nm}$ Py film by a field-pump x-ray-probe experiment and studied the precessional damping of the 
$\mathrm{Fe}$ and $\mathrm{Ni}$ magnetic moments separately within the alloy as a function of applied field. Furthermore, we have investigated the temperature dependence of the damping constant.

\section{Experimental procedures}

For our pump-probe experiments we use a stripline geometry to generate a magnetic field pulse at the sample region. For reflection geometry experiments the stripline design is simpler than a coplanar waveguide structure used in FMR, and provides the possiblity to investigate the magnetization dynamics even in samples with very thick and complex layer structures or samples on a non-transparent substrate. A $7 \times 7 \mathrm{~mm}^{2}$ Si substrate supports a $350 \mu \mathrm{m}$ wide stripline structure, consisting of a triple stack $(\mathrm{Cu}(75 \mathrm{~nm}) / \mathrm{Py}(25 \mathrm{~nm}) / \mathrm{Cu}(3 \mathrm{~nm}))$ prepared by ion beam sputtering through a mask. The HV sputtering chamber has a base pressure of $2 \times 10^{-9}$ mbar and the Ar sputter gas has a partial pressure of $4 \times 10^{-4}$ mbar during deposition. The use of Si with a naturally formed oxide layer on its top prevents any substrate-induced in-plane anisotropy. The $75 \mathrm{~nm}$ thick $\mathrm{Cu}$ layer serves as main conducting layer, whereas the second $\mathrm{Cu}$ layer prevents the oxidation of the magnetic system. The stripline is connected to an electronic pulse generator providing voltage pulses of $10 \mathrm{~V}$ amplitude and $10 \mathrm{~ns}$ width to excite the magnetic system. The voltage pulse is directly converted into an Oersted field pulse $H_{p}$ that is perpendicular to the stripline. A bias field $H_{b}$ can be applied parallel to the stripline in order to align the initial magnetization prior to excitation.

For the experiments we used the ALICE chamber housing an x-ray diffractometer [17] as the end station of the undulator beamline UE52-SGM at the Bessy synchrotron of the Helmholtz-Zentrum Berlin (HZB). The beamline provides photons with variable polarization and energy especially in the range covering the $L_{3,2}$ edges of the $3 \mathrm{~d}$ transition metal elements. Therefore it is ideally suited for magnetic studies, be it absorption measurements, reflectivity studies [18] or holography experiments [19, 20]. During single bunch operation mode the repetition frequency of the electron bunches is $1.25 \mathrm{MHz}$ with a pulse length of $50 \mathrm{ps}$, enabling pump-probe type experiments for processes much faster than $800 \mathrm{~ns}$, i.e. the time between two photon pulses. Temporal resolution is then achieved by electronically shifting the voltage pulse with respect to a trigger signal from the storage ring. With our system steps as small as 10 ps are possible which is well below the intrinsic probe pulse length. The reflected intensity is detected by a photodiode current and monitored as a function of delay time between pump and probe. In order to obtain reasonable statistics, we average over about $10^{7}$ excitations, assuming that the dynamics of the system is repetitive and completely reversible.

In figure 1 a schematic of the experimental setup for time-resolved measurements is shown. The stripline is oriented perpendicular to the scattering plane along the $y$ direction, a pair of Helmholtz coils provides the bias field $H_{b}$ parallel to the stripline. The whole assembly together with hf wiring is vacuum compatible and mounted on the 


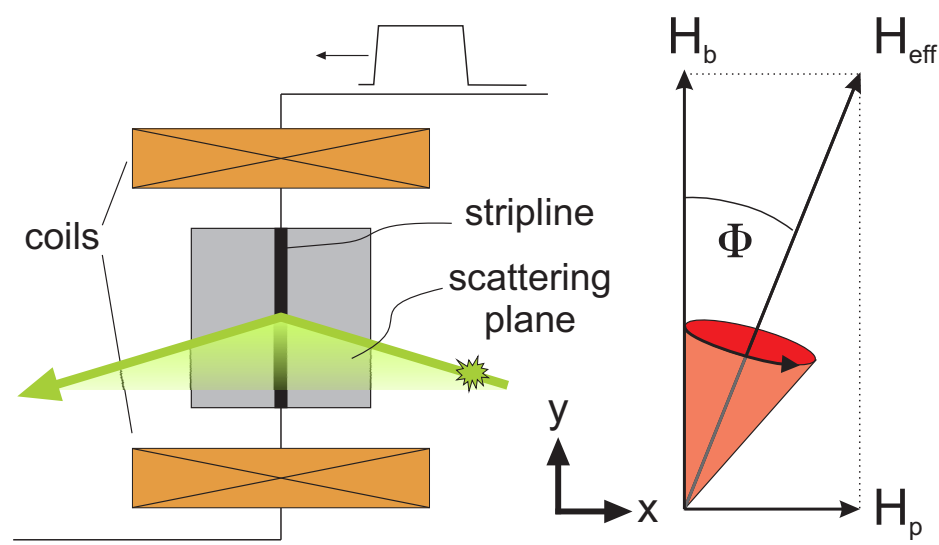

Figure 1. Schematic of the setup and fields applied at the sample region. The $350 \mu \mathrm{m}$ stripline is centered on a $\mathrm{Si}$ substrate and oriented perpendicular to the scattering plane. A pair of coils provides the bias field $H_{b}$ along the $y$-axis, the pulse field from the stripline is parallel to the $x$-axis. With our setup the change in the $M_{x^{-}}$component is measured while the magnetization precesses around the effective field direction $H_{\text {eff. }}$.

cold finger of a cryostat in the ALICE chamber. The bias field $H_{b}$ is used to saturate the sample and align the magnetization prior to excitation and thus defines the initial condition. An electromagnet used to apply a field parallel to the surface along the $x$-direction is part of the ALICE chamber and not shown explicitly in figure 1.

The diffractometer environment enables q-dependent studies, and any incident angle desired can be used. In general, the magnetic contribution to the scattered intensity is $I_{m} \propto \sigma \cdot \mathbf{M}$, where $\sigma$ describes the photon helicity and $M$ is the magnetization vector. In reflection geometry at small incident angles we therefore monitor the evolution of the in-plane magnetization component $M_{x}$. The $M_{z^{-}}$or out-of-plane component is usually very small due to the strong in-plane anisotropy, and on top of that the sensitivity to this component is very weak. For these reasons also $M_{z}$ can be neglected, and the magnetic scattered intensity can be written as $I_{m}=I_{m}^{0} \cdot \sin \Phi$. Here $I_{m}^{0}$ is the absolute magnetic signal at a given incident angle and photon energy, and $\Phi$ is the angle between the magnetization $M$ and the $y$-axis.

One of the challenges for time-resolved experiments is to ensure the relative position between the sample and the beam, especially when cooling the sample. On the one hand, the feedback loop of the synchrotron might slightly change the beam with respect to the sample position. On the other hand, the sample sitting at the end of a long cold finger changes its position as a function of temperature. These experimental details are negligible for extended samples, but become important for narrow striplines and the more so the narrower the stripline is. For our case, with a $350 \mu \mathrm{m}$ wide stripline we succeeded to observe a clear time resolved signal after a thorough temperature stabilization. 


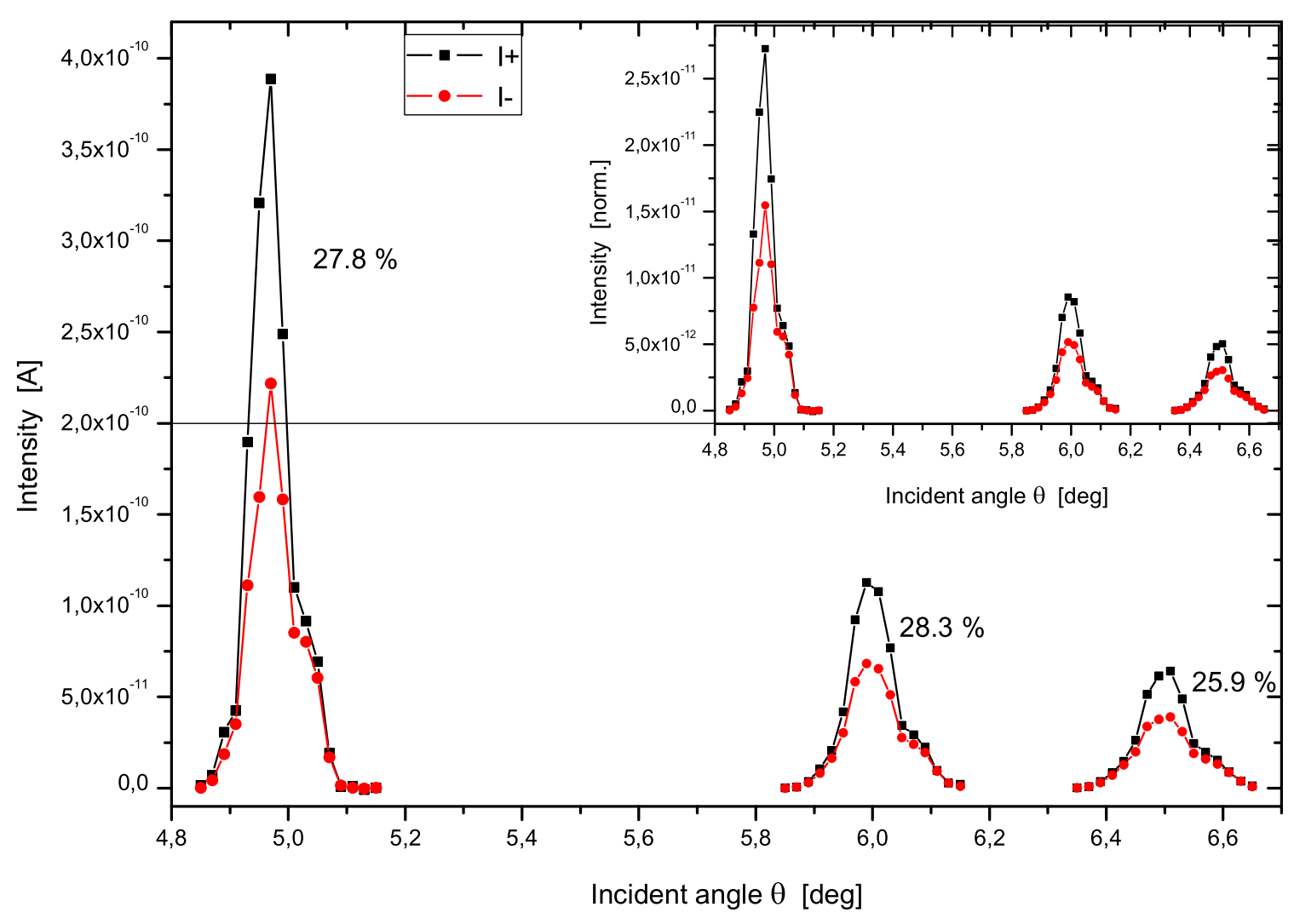

Figure 2. Rocking scans with opposite field directions $\left(I^{+}\right.$and $\left.I^{-}\right)$to determine the asymmetry at each incident angle. The main graph shows the photocurrent as measured together with a horizontal line indicating the measuring range of the ammeter in order to obtain low-noise data in the incoming signal, the inset shows the normalized data to show that the difference between $I^{+}$and $I^{-}$is not an effect due to the ring decay.

\section{Results and Discussion}

\subsection{Static experiments}

For an optimized XRMS signal for both the Fe and Ni resonant edges, we first characterized the static magnetic properties of the Py film. Rocking scans where measured at various positions on the reflectivity curve to define a proper angle of incidence. Figure 2 shows these scans performed at various incident angles; a field of 275 Oe was applied along the $x$-axis to saturate the sample in the two opposite directions $\left(I^{+}, I^{-}\right)$, and the difference between the two signals is of magnetic origin. The main graph shows the photocurrent as measured, whereas in the inset these data are normalized to the ring current. A strong decay of the intensity is observed as the incident angle is increased, while the resulting magnetic asymmetry $\left(I^{+}-I^{-}\right) /\left(I^{+}+I^{-}\right)$is more or less the same with $27.8,28.3$ and $25.9 \%$ at 5.0, 6.0 and $6.5^{\circ}$, respectively. For angles larger than $6.5^{\circ}$ both the overall signal and the asymmetry are further reduced. For the ammeter used in this experiment, the horizontal line indicates the limit of the measurement range 

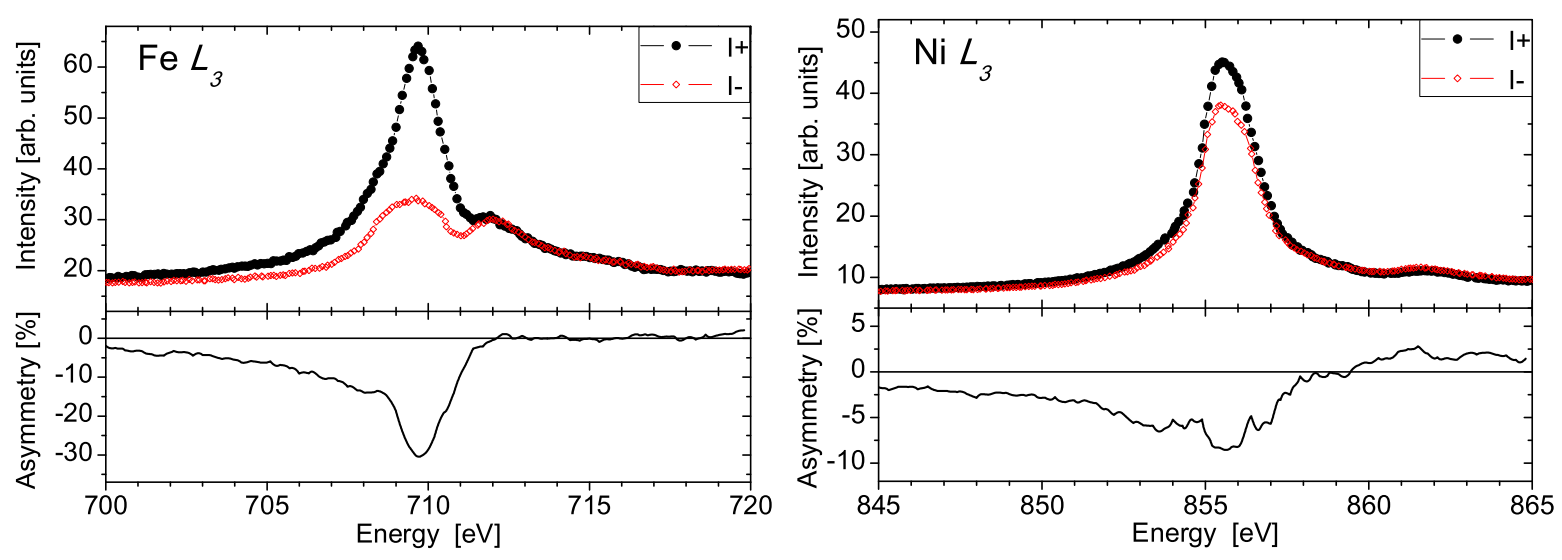

Figure 3. The upper panels show energy scans with circularly polarized photons at an incident angle of $6^{\circ}$ around the $\mathrm{Fe}$ and Ni $L_{3}$ edges, performed for two oppositely applied magnetic fields along the $x$-axis (see figure 1 ). The lower panels show the resulting magnetic asymmetry.

$\left(2 \times 10^{-10} \mathrm{~A}\right)$. Below this level the background noise from the ammeter is almost two orders of magnitude smaller than for photocurrents exceeding this value. Therefore an angle of incidence of $6.0^{\circ}$ is the best compromise between a strong absolute and magnetic signal and a minimal detector noise level even for the highest photon flux immediately after an injection.

During alignment photon energy values close to the nominal absorption edges were used. To enhance the magnetic signal for the time-resolved experiments, the photon energy was then refined at the given incident angle. The stripline sample was saturated in-plane along the $x$-direction, and the photon energy was swept through the resonance while keeping the circular polarization constant. The result of these energy scans for positive and negative saturation $\left(I^{+}\right.$and $I^{-}$, respectively) are shown for both elements in figure 3. The lower panels show the asymmetry which is the magnetic contrast at the respective energies. We concentrated on the $L_{3}$ edges, where the magnetic contrast is larger in comparison to the $L_{2}$ edges. In contrast to XMCD data, the energy scans carried out using XRMS contain both magnetic and charge contributions and therefore the shape strongly depends on the incident angle.

For a static characterization of the sample we measured hysteresis loops as shown in figure 4. As discussed above, the magnetic contribution solely depends on the angle $\Phi$ for a given incident angle and photon energy. For a hysteresis loop along the $x$-direction, positive and negative saturation are then equivalent to $\Phi= \pm 90^{\circ}$, respectively. A net magnetization zero corresponds to an alignment along the $y$-axis, and is normalized to unity as this is the reference situation prior to any excitation. The detected intensity change during a time-resolved scan can thereby be converted into the opening angle $\Phi$ via the hysteresis loop data using $I_{m}=I_{m}^{0} \cdot \sin \Phi$. 


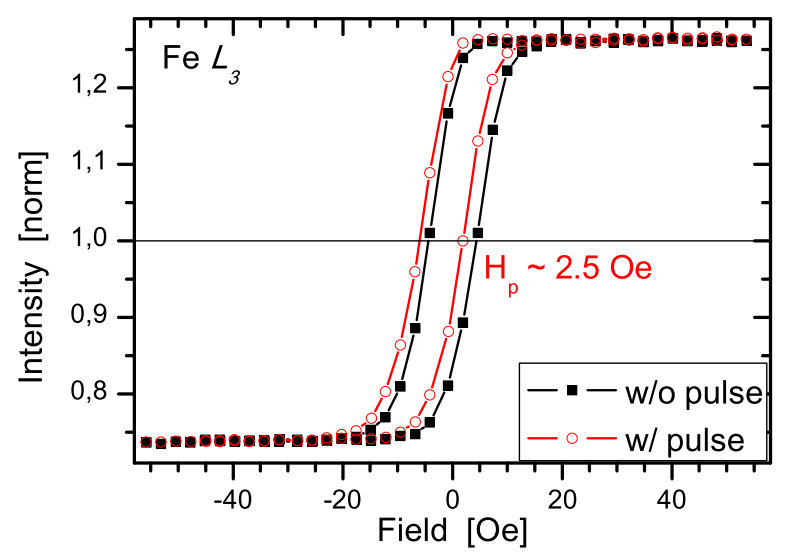

Figure 4. Hysteresis loop measured at the Fe $L_{3}$ edge, with (open) and without (full symbols) applying a field pulse to the sample. A shift of 2.5 Oe is observed. The overall hard-axis behavior indicates a small anisotropy with its easy axis along the stripline direction.

To determine the amplitude of the Oersted field from the stripline we compare $\mathrm{x}$ ray recorded hysteresis loops with and without pulsed excitation. Figure 4 shows data measured at the Fe edge: In both cases we have a coercivity of 4.5 Oe and only a weak anisotropy, but when the current pulse is introduced the whole hysteresis loop is shifted due to the additonal field $H_{p}$ along the $x$-axis. From this shift we can estimate the pulsed field to be in the order of +2.5 Oe, oriented parallel to the positive direction of the applied external field (i.e. in $x$-direction). Another method to quantify $H_{p}$ from the dynamic results is shown in the next section.

\subsection{Time-resolved experiments}

For the time-resolved experiments the initial magnetization is aligned with the bias field $H_{b}$ along the stripline ( $y$-direction). There is no additional field applied in the $x$-direction. Upon the field pulse, we observe a time dependent change in intensity, implying that $M_{x}$ (x-component of the magnetization) changes in time. The intensity changes are then converted into opening angles $\Phi$ and plotted as a function of delay time to monitor the magnetization dynamics in the film.

To compare the magnetization dynamics of the Fe and Ni magnetic moments, we have plotted both signals in figure 5 for a set of bias fields. For each bias field, the photon energy was set to the Fe (full symbols) and $\mathrm{Ni}$ (open symbols) $L_{3}$-edges, and we followed the intensity change as a function of time. All curves show an initial rise followed by a damped precessional oscillation, as expected for step-like field-pulse excitations. Two main features are clearly visible in this data set: with the increase of bias field, the opening angle decreases, and at the same time the frequency increases. The initial slope $\frac{\mathrm{d} \phi}{\mathrm{d} t}$ is the same for all bias field values. This feature leads to a decrease in the response time, i.e. the time between the onset of the current pulse and the first transition through the new equilibrium direction. 


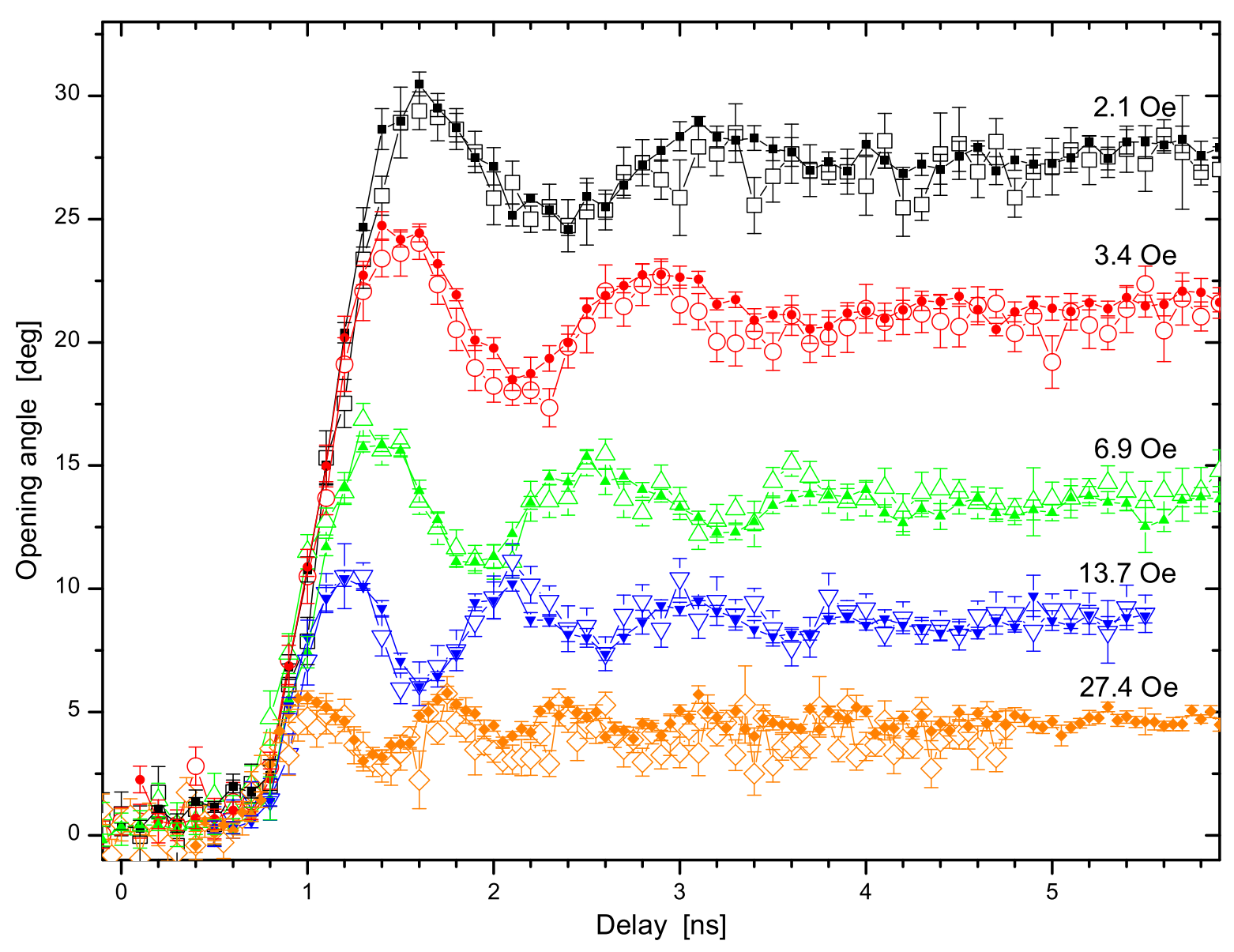

Figure 5. Comparison of the magnetization dynamics measured at the Fe (full) and $\mathrm{Ni}$ (open symbols) resonant edges for a set of different bias fields. The detected intensity is converted into opening angle $\Phi$ according to the hysteresis curves.

The data presented in figure 5 clearly show the same oscillation amplitude, frequency and phase for both $\mathrm{Ni}$ and Fe magnetic moments over the entire range of delay time. This is expected as the exchange coupling between the Fe and Ni moments is rather strong and confirms the observation by Bailey et al. as well [10]. The larger error bars for the $\mathrm{Ni}$ in comparison with the Fe data arise for two reasons: The asymmetry at the $\mathrm{Ni}$ edge is smaller by a factor of almost four, leading to an increase of the statistical errors in case of identical counting times. Second, for identical glancing angle the scattering vector for scanning the $\mathrm{Ni}$ edge is higher because of the shorter wavelength, implying that the reflectivity from the Py film is reduced.

In the following we concentrate on the data obtained at the Fe edge for further data analysis. We use a damped sinusoidal oscillation to describe the magnetic response following the field pulse:

$$
\Phi(t)=\Phi_{0}+\Phi^{\prime} \exp \left(-t / t_{0}\right) \cdot \sin (\omega t+\varphi)
$$

Here $\Phi_{0}$ is the opening angle at equilibrium after the oscillations are damped out, and $\Phi^{\prime}$ is the oscillation amplitude. The precessional motion is described by the decay time $t_{0}$ 


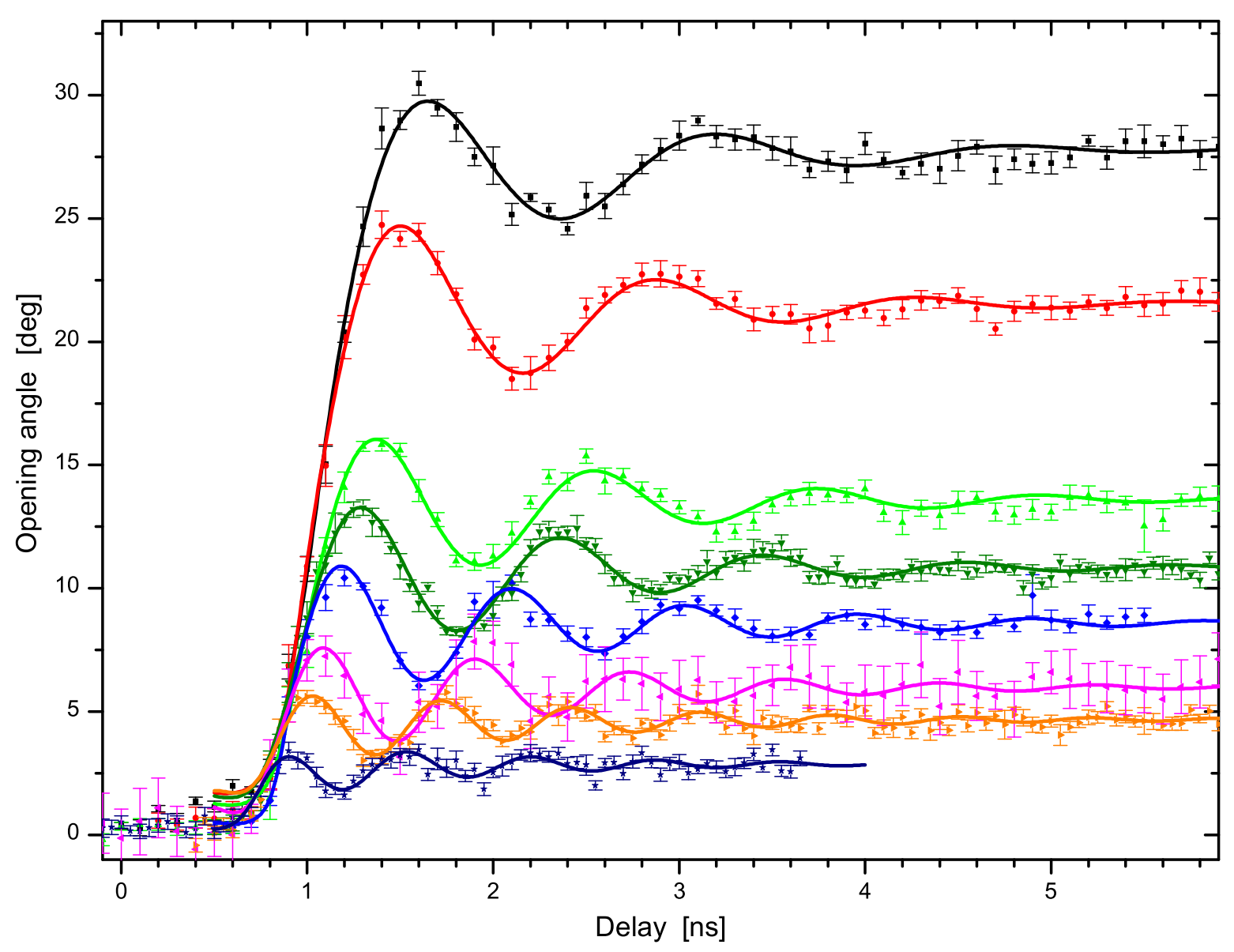

Figure 6. Fit results for data obtained at the Fe edge using the damped sinusoidal function described in the text.

and angular frequency $\omega=2 \pi f$. Silva et al. have shown that this procedure gives results that are very similar to full LLG simulations [21]. In figure 6 the resulting fits are shown together with the data. The overall change in the magnetization direction assumes values of almost $\Phi=30^{\circ}$ in the case of small bias fields. However, the precession itself is always restricted to $\pm 2^{\circ}$ around the new equilibrium direction, thus the approximative description for small angle precession is appropriate. In FMR experiments, precessional cones exeeding few degrees are considered to be large and lead to the Suhl instability [22] where spin waves are supposed to be generated and reduce the absolute value of the magnetization. This is because in FMR experiments an rf-field is constantly applied to the sample leading to a forced precession of the sample magnetization. In a pumpprobe experiment, however, the excitation is given by a single field pulse which merely initiates a free precessional motion of magnetization. In similar MOKE experiments no generation of spin waves - manifesting themselves in a significant reduction of the absolute value of the magnetization - has been observed, despite excitation angles close to the switching regime [15].

Figure 5 shows that after about 4 ns the oscillations are damped out and the new equilibrium direction of the magnetization given by $\Phi_{0}$ is reached. The latter 

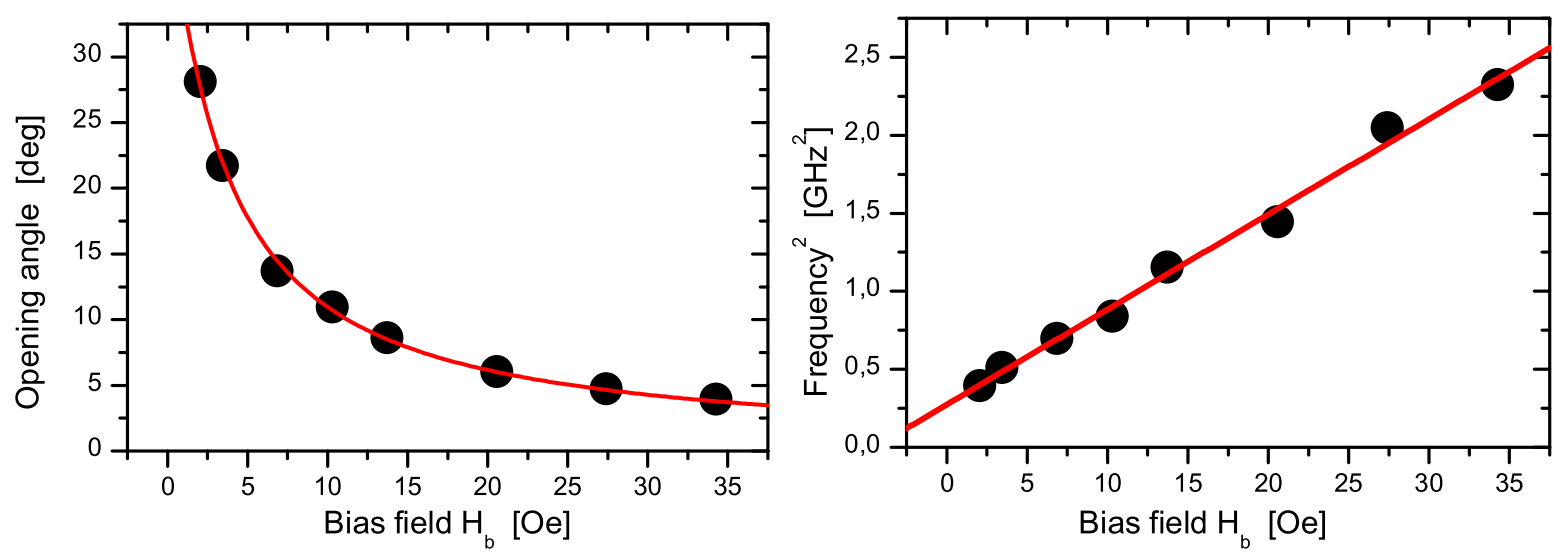

Figure 7. In (a) the opening angle is shown as a function of bias field $H_{b}$, the red line is a fit with the values $H_{p}$ and $H_{k}$ as free parameters, see text. In (b) the square of the frequency is plotted as a function of bias field $H_{b}$, with a linear fit according to the Kittel formula.

one is given by minimizing the free energy of the system, and leads to the condition $H_{k} \cdot \sin \Phi_{0}-H_{p}+H_{b} \cdot \tan \Phi_{0}=0$. Even for the largest angle measured, we may assume $\sin \Phi \approx \tan \Phi$ within an acceptable error bar. In this way we can use the dependence of the opening angle on the bias field to again determine the pulsed field $H_{p}$ : figure $7 \mathrm{a}$ shows the change of the opening angle with varying bias field, where the solid line is a fit according to $\tan \Phi=H_{p} /\left(H_{b}+H_{k}\right)$. $H_{k}$ accounts for the additional anisotropy along the stripline that is observed in hysteresis loops measured in both $x$ - and $y$-directions with MOKE. From the fit we obtain 2.4 $( \pm 0.6)$ Oe for the magnitude of the pulsed field $H_{p}$ which is in very good agreement with what we deduced from our prior hysteresis shift test. The anisotropy field is $2.6( \pm 0.2)$ Oe and presumably induced in the growth process. The anisotropy is in accordance with prior MOKE data.

In Py the saturation magnetization $M_{s}$ is much larger than $H_{b}+H_{k}$ and therefore the frequency dependence can be well described by the Kittel formula $\omega^{2}=$ $\left(\gamma \mu_{0}\right)^{2} \cdot\left[H_{\text {eff }}\left(H_{\text {eff }}+M_{s}\right)\right][23]$, where $\gamma$ is the gyromagnetic ratio, $\mu_{0}$ the vacuum permeability, and $\omega$ the angular frequency of the precession. In our case the effective field is a combination of the uniaxial anisotropy as calculated from the fit in figure $7 \mathrm{a}$, and the applied and pulsed field at the sample. The calculations give $H_{\text {eff }} \ll M_{s}$, and $H_{p} \ll H_{b}$. Therefore the above equation can be simplified into $\omega^{2}=\left(\gamma \mu_{0}\right)^{2} \cdot\left[\left(H_{b}+H_{k}\right) M_{s}\right]$ and can be used for a linear fit of the frequency dependence as shown in Figure 7b. Assuming a gyroscopic splitting factor $g=2$ for Fe, we obtain $M_{s}=619( \pm 13) \mathrm{kA} / \mathrm{m}$, which is in good agreement with prior SQUID measurements yielding $M_{s}=570 \mathrm{kA} / \mathrm{m}$. The anisotropy field deduced from the dynamic measurements is $4.5( \pm 0.3)$ Oe, which is larger by a factor of 1.7 than the static result obtained from the opening angle. This difference can be attributed to an additional dynamic anisotropy contribution as described earlier [24]. In turn if we take the SQUID result as value for $M_{s}$, the resulting splitting factor is $g=2.08( \pm 0.04)$, which is in very good accordance with literature values [14]. 


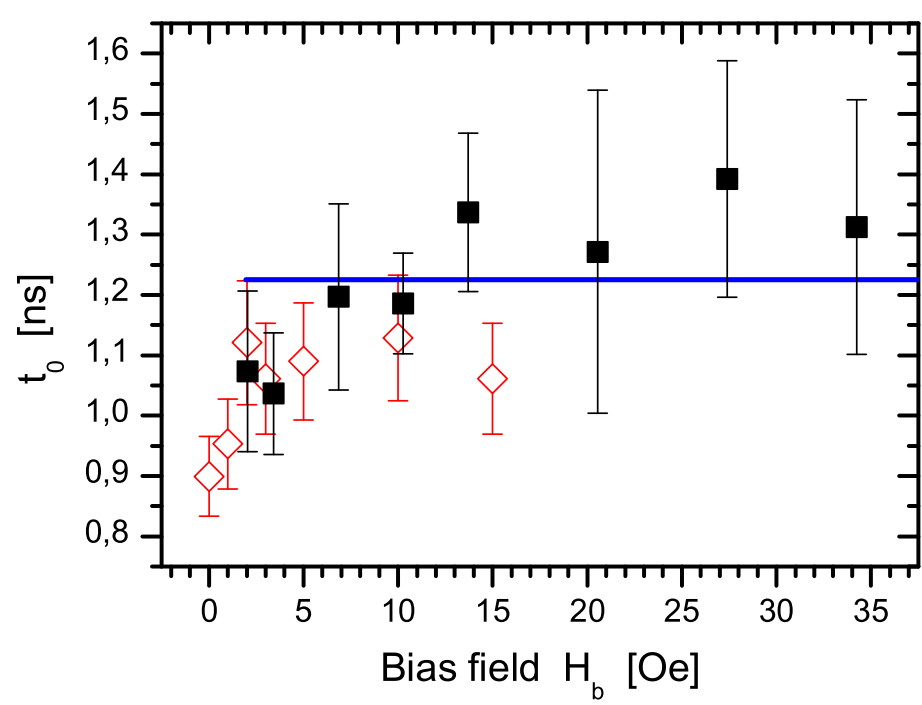

\begin{tabular}{|r|r|r|}
\hline$H_{b}(\mathrm{Oe})$ & $t_{0}(\mathrm{~ns})$ & $\Delta t_{0}(\mathrm{~ns})$ \\
\hline 2,1 & 1,07 & 0,13 \\
3,4 & 1,04 & 0,10 \\
6,9 & 1,20 & 0,15 \\
10,3 & 1,19 & 0,08 \\
13,7 & 1,34 & 0,13 \\
20,6 & 1,27 & 0,27 \\
27,4 & 1,39 & 0,20 \\
34,3 & 1,31 & 0,21 \\
\hline
\end{tabular}

Figure 8. The full symbols show the damping constants from the fits to the data (figure 6) as a function of bias field. The open symbols show values obtained with a MOKE setup using a pulsed field of 3 Oe and are extracted from [21].

From the fits in Figure 6, we also obtain a characteristic decay time $t_{0}$ for the damping of the precession. Figure 8 gives an overview on decay times determined from all pulsed measurements, with the blue horizontal line marking the averaged value. There is a tendency towards increased damping (i.e. smaller $t_{0}$ ) for small bias fields. This tendency has been pointed out by Nibarger et al. [25] who carried out tr-MOKE experiments with similar settings. The large error bars result from the small number of oscillations that can be recorded for our sample. Enhancing the number of oscillations should reduce the error bar, but is challenging to achieve with our setup: Increasing the bias field does not reduce the error bars, as the reduced signal amplitude does not qualitatively improve the data. On the other hand, for the two smallest bias field values the signal amplitudes are sizeable, but the decay times are short compared to the time of one oscillation, which again does not improve the error bars. In general, we note the decay times from tr-XRMS for elemental Fe moments in Py agree rather well with those obtained by tr-MOKE measurements averaging over all constituting elements on this time scale.

\subsection{Low temperature experiments}

The ALICE chamber is equipped with a continuous flow cryostat which was operated with liquid Nitrogen (LN2) for our experiments. In Figure 9 we compare results taken for two different bias fields at $150 \mathrm{~K}, 300 \mathrm{~K}$, and $350 \mathrm{~K}$. These data confirm that the necessary stability can be reached with our setup to conduct time-resolved scans at various temperatures. At the same time we do not observe significant changes in the angle, frequency and in the damping behavior within the temperature range $150-350 \mathrm{~K}$. The increased error bars indicate the afore-mentioned challenges with the temperature 


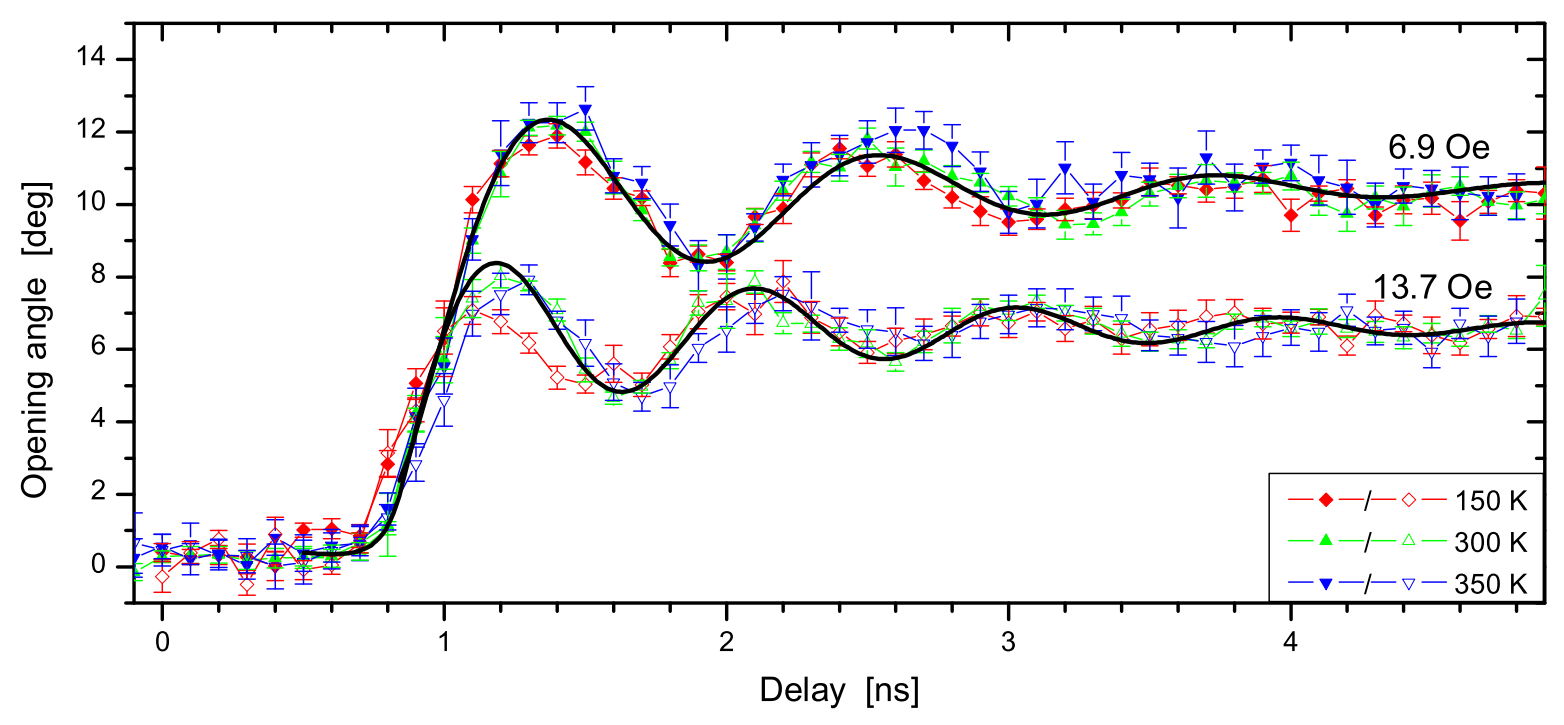

Figure 9. Delay scans for two different bias fields and at three different temperatures. The black line is the best fit to the $300 \mathrm{~K}$ data. For the results obtained at $150 \mathrm{~K}$ the onset of the pulse is hard to define and may lead to a small phase shift on the time-axis.

feedback loop. This result is in accordance with FMR results on Fe and Ni films in the high frequency regime obtained by Patton and Wilts [26], where the damping was found to be constant within the temperature range considered here, and with more recent FMR results on Py by Sierra et al. using a Vector Network Analyzer [27], which also match the low frequency range. In both references, an increase in damping is observed below about $120 \mathrm{~K}$. Future experiments will focus on systems that significantly change their properties with temperature, e.g. a metal-insulator transition system adjacent to the Py layer. Then the additional effect of the metallic phase as a spin sink might be separated from a pure interface-related damping that is also present for the insulating phase.

\section{Conclusion}

We have observed element-resolved precessional dynamics in a $25 \mathrm{~nm}$ thick $\mathrm{Fe}_{20} \mathrm{Ni}_{80}$ film shaped in form of a stripline with a width of $350 \mu \mathrm{m}$. A step-like field-pulse excitation in combination with pulsed circularly polarized x-rays is used for this pumpprobe experiment. Both $\mathrm{Fe}$ and $\mathrm{Ni}$ elemental moments precess at the same frequency and are aligned parallel. The damping determined from x-ray detected magnetization dynamics is in good accordance with MOKE results from literature averaging over both elements. The decay time is about $1.2 \mathrm{~ns}$, with a tendency to decrease with decreasing precessional amplitude. The temperature dependence of the precessional dynamics was found to be negligible in the range $150 \mathrm{~K}$ to $350 \mathrm{~K}$.

The presented data show the feasibility to conduct magnetization dynamics studies in thin films with an element-selective probe with a temporal resolution limited by 
the photon pulse length of $50 \mathrm{ps}$. In this way magnetic multilayer structures containing different magnetic elements can directly be accessed in the low-GHz regime. The shorter wavelength and larger penetration depth of soft x-rays in comparison to visible light used in MOKE experiments enable to also reach deeply buried layers, whereas the temporal resolution available with modern fs-Laser systems cannot be achieved with the synchrotron-based technique. The combination of the time-resolved setup with a diffractometer environment allows the observation at various scattering vectors, and therefore to separate interface and bulk magnetization properties. With the pumpprobe technique we access the regime of free precessional motion, which is not limited to small excitation angles and renders the investigation of large angle magnetization dynamics posssible, like it is the case in switching dynamics. Thus we circumvent the occurrence of the Suhl instability that limits typical resonant excitation experiments to a few degrees in opening angle [28]. Future experiments will focus on multilayer systems with emphasis on interlayer and interface effects.

\section{Acknowledgements}

We would like to thank Denise Erb for her support during the measurements at Bessy II, Berlin, and Min-Sang Lee for valuable comments about the manuscript. This work was financially supported by the BMBF Verbundforschung (05KS7PC1), which is gratefully acknowledged. We are also thankful to the HZB Berlin for travel support under BMBF $05 \mathrm{ES} 3 \mathrm{XBA} / 5$. 


\section{References}

[1] J. Miltat and A. Thiaville. Magnets fast and small. Science, 290(5491):466-467, 2000.

[2] M. Bauer, R. Lopusnik, J. Fassbender, and B. Hillebrands. Suppression of magnetic-field pulseinduced magnetization precession by pulse tailoring. Applied Physics Letters, 76(19):2758 -2760, may. 2000.

[3] Th. Gerrits, H. A. M. van den Berg, J. Hohlfeld, L. Bar, and Th. Rasing. Ultrafast precessional magnetization reversal by picosecond magnetic field pulse shaping. Nature, 418(6897):509-512, August 2002.

[4] I. Tudosa, C. Stamm, A. B. Kashuba, F. King, H. C. Siegmann, J. Stohr, G. Ju, B. Lu, and D. Weller. The ultimate speed of magnetic switching in granular recording media. Nature, 428(6985):831-833, April 2004.

[5] O. Hellwig, T. Hauet, T. Thomson, E. Dobisz, J. D. Risner-Jamtgaard, D. Yaney, B. D. Terris, and E. E. Fullerton. Coercivity tuning in $\mathrm{Co} / \mathrm{Pd}$ multilayer based bit patterned media. Applied Physics Letters, 95(23):232505, 2009.

[6] S. S. P. Parkin, M. Hayashi, and L. Thomas. Magnetic Domain-Wall Racetrack Memory. Science, 320(5873):190-194, 2008.

[7] M. Farle. Ferromagnetic resonance of ultrathin metallic layers. Reports on Progress in Physics, 61(7):755, 1998.

[8] D. A. Arena, E. Vescovo, C.-C. Kao, Y. Guan, and W. E. Bailey. Combined time-resolved xray magnetic circular dichroism and ferromagnetic resonance studies of magnetic alloys and multilayers (invited). Journal of Applied Physics, 101(9):09C109, 2007.

[9] G. Boero, S. Rusponi, P. Bencok, R. Meckenstock, J.-U. Thiele, F. Nolting, and P. Gambardella. Double-resonant x-ray and microwave absorption: Atomic spectroscopy of precessional orbital and spin dynamics. Phys. Rev. B, 79(22):224425, Jun 2009.

[10] W. E. Bailey, L. Cheng, D. J. Keavney, C.-C. Kao, E. Vescovo, and D. A. Arena. Precessional dynamics of elemental moments in a ferromagnetic alloy. Physical Review B, 70(17):172403, Nov 2004.

[11] T. Martin, G. Woltersdorf, C. Stamm, H. A. Dürr, R. Mattheis, C. H. Back, and G. Bayreuther. Layer resolved magnetization dynamics in interlayer exchange coupled $\mathrm{Ni}_{81} \mathrm{Fe}_{19} / \mathrm{Ru} / \mathrm{Co}_{90} \mathrm{Fe}_{10}$ by time resolved x-ray magnetic circular dichroism. Journal of Applied Physics, 103(7):07B112, 2008.

[12] Y. Guan, W. E. Bailey, C.-C. Kao, E. Vescovo, and D. A. Arena. Comparison of time-resolved x-ray magnetic circular dichroism measurements in reflection and transmission for layer-specific precessional dynamics measurements. Journal of Applied Physics, 99(8):08J305, 2006.

[13] S. Buschhorn, F. Brüssing, R. Abrudan, and H. Zabel. Time resolved x-ray resonant magnetic scattering in reflection geometry. Journal of Synchrotron Radiation. accepted.

[14] J. P. Nibarger, R. Lopusnik, Z. Celinski, and T. J. Silva. Variation of magnetization and the Landé g factor with thickness in Ni-Fe films. Applied Physics Letters, 83(1):93-95, 2003.

[15] Th. Gerrits, T. J. Silva, J. P. Nibarger, and Th. Rasing. Large-angle magnetization dynamics investigated by vector-resolved magnetization-induced optical second-harmonic generation. Journal of Applied Physics, 96(11):6023-6028, 2004.

[16] T. J. Silva, P. Kabos, and M. R. Pufall. Detection of coherent and incoherent spin dynamics during the magnetic switching process using vector-resolved nonlinear magneto-optics. Applied Physics Letters, 81(12):2205-2207, 2002.

[17] J. Grabis, A. Nefedov, and H. Zabel. Diffractometer for soft x-ray resonant magnetic scattering. Review of Scientific Instruments, 74(9):4048-4051, 2003.

[18] A. Bergmann, J. Grabis, A. Nefedov, K. Westerholt, and H. Zabel. X-ray resonant magnetic scattering study of [Co $2 \mathrm{MnGe} / \mathrm{Au}] \mathrm{n}$ and [Co $2 \mathrm{MnGe} / \mathrm{V}] \mathrm{n}$ multilayers. Journal of Physics D: Applied Physics, 39(5):842, 2006.

[19] O. Hellwig, S. Eisebitt, W. Eberhardt, W. F. Schlotter, J. Lüning, and J. Stöhr. Magnetic imaging 
with soft x-ray spectroholography. Journal of Applied Physics, 99(8):08H307, 2006.

[20] C. M. Günther, O. Hellwig, A. Menzel, B. Pfau, F. Radu, D. Makarov, M. Albrecht, A. Goncharov, T. Schrefl, W. F. Schlotter, R. Rick, J. Lüning, and S. Eisebitt. Microscopic reversal behavior of magnetically capped nanospheres. Phys. Rev. B, 81(6):064411, Feb 2010.

[21] T. M. Crawford, T. J. Silva, C. W. Teplin, and C. T. Rogers. Subnanosecond magnetization dynamics measured by the second-harmonic magneto-optic kerr effect. Applied Physics Letters, 74(22):3386-3388, 1999.

[22] P. W. Anderson and H. Suhl. Instability in the motion of ferromagnets at high microwave power levels. Phys. Rev., 100(6):1788-1789, Dec 1955.

[23] C. Kittel. Introduction to Solid State Physics. Wiley, New York, 1996.

[24] R. Lopusnik, J. P. Nibarger, T. J. Silva, and Z. Celinski. Different dynamic and static magnetic anisotropy in thin Permalloy films. Applied Physics Letters, 83(1):96-98, 2003.

[25] J. P. Nibarger, R. Lopusnik, and T. J. Silva. Damping as a function of pulsed field amplitude and bias field in thin film Permalloy. Applied Physics Letters, 82(13):2112-2114, 2003.

[26] C. E. Patton and C. H. Wilts. Temperature dependence of the Ferromagnetic Resonance linewidth in thin NiFe films. Journal of Applied Physics, 38(9):3537-3540, 1967.

[27] J. F. Sierra, V. V. Pryadun, F. G. Aliev, S. E. Russek, M. García-Hernández, E. Snoeck, and V. V. Metlushko. Temperature dependent dynamic and static magnetic response in magnetic tunnel junctions with Permalloy layers. Applied Physics Letters, 93(17):172510, 2008.

[28] H. Suhl. The theory of ferromagnetic resonance at high signal powers. Journal of Physics and Chemistry of Solids, 1(4):209 - 227, 1957. 